\title{
Flexible Molecular-Scale Electronic Devices Composed of Diarylethene Photoswitching Molecules
}

\author{
Dongku Kim, Hyunhak Jeong, Hanki Lee, Wang-Taek Hwang, Jannic Wolf, Elke Scheer, \\ Thomas Huhn, Heejun Jeong, and Takhee Lee*
}

Since a conceptual model for a molecular rectifier was first proposed in $1974,^{[1]}$ molecular electronic devices, which utilize molecules as electronic device components, have been widely studied for use in rectifiers, transistors, switches, and memories. ${ }^{[2-11]}$ However, any functional molecular monolayer devices are hardly practical because a typical vertically structured molecular device has a very low device yield $(<\sim 1 \%)$ owing to electrical shorts that may occur as a result of the top electrode's penetration through the thin molecular layers. ${ }^{[12-14]}$ Single-molecule devices, however, are prone to large fluctuations of their properties due to lack of control of positioning the molecules with atomic precision into electrodes and are suffering from the low conductance of these devices. To overcome this problem, several ideas have been proposed, such as using an intermediate protective layer composed of a conducting polymer, ${ }^{[15-18]}$ or graphene film ${ }^{[19-21]}$ between the molecular layer and the top electrode. These approaches enhanced the device yield of the molecular devices tremendously (>90\%). ${ }^{[19]}$

With the development of high-yield molecular devices, it became possible to fabricate them on plastic flexible substrates and study their electrical properties when subjected to mechanical deformation, which would not be possible with low-yield molecular devices. Previously, we demonstrated flexible molecular-scale devices made with alkanethiols. ${ }^{[18]}$ However, alkanethiol molecular devices were simply insulating due to the large molecular orbital energy gap ${ }^{[12,13,22-27]}$ and are not suitable for industrial applications due to the absence of any potential device functionality. Subsequently, we applied ferrocene-alkanethiols, known as

D. Kim, H. Jeong, H. Lee, W.-T. Hwang, Prof. T. Lee

Department of Physics and Astronomy

and Institute of Applied Physics

Seoul National University

Seoul 151-747, Korea

E-mail: tlee@snu.ac.kr

Dr. J. Wolf, ${ }^{[+]}$Dr. T. Huhn

Department of Chemistry

University of Konstanz

78457, Konstanz, Germany

Prof. E. Scheer

Department of Physics

University of Konstanz

78457, Konstanz, Germany

Prof. H. Jeong

Department of Applied Physics

Hanyang University

Ansan 426-791, Korea

${ }^{[+]}$Photovoltaics and Solar Engineering department, King Abdullah University of Science and Technology, Thuwal 23955-6900, Saudi Arabia rectifying molecules, ${ }^{[8,28-32]}$ to flexible molecular devices. Unfortunately, the results showed that a more sophisticated design would have been necessary to fully realize their functionality. ${ }^{[33]}$ Therefore, it was decided to attempt to demonstrate applying truly functional molecules to flexible molecular-scale devices.

In this study, we fabricated and characterized flexible molecular-scale electronic devices made with photoswitching molecules of the diarylethene class on a flexible plastic substrate. For other species of this class, two electrical conductance (high or low conductance) states were found in different device architectures and were controlled by exposure to ultraviolet (UV) or visible light. ${ }^{[6,34-36]}$ In particular, we investigated the reliability of the electronic characteristics for both states of the diarylethene flexible molecular devices under flat substrate condition and various substrate bending conditions, such as different bending radii $(10,5$, and $1 \mathrm{~mm}$ ) or repeated bending cycles (up to 1000). We also examined the electrical conduction mechanism behind the two photoswitching states using temperature-variable current-voltage characterization.

Figure 1(a) shows the schematics illustrating the fabrication processes for the molecular electronic devices on a flexible substrate described in this study. First, Au (60 nm thick)/ Ti (5 nm thick) was deposited on a polyimide (PI) substrate (Neopulim L-3430 purchased from Mitsubishi Gas Chemical Co., Inc.) using a shadow mask. The $\mathrm{Au} / \mathrm{Ti}$ film was deposited at a slow deposition rate of $\sim 0.1 \AA / \mathrm{s}$ with an electron-beam evaporator. Then, insulating photoresist (PR; Az5214E purchased from Az Electronic Materials) was spin-coated onto the surface and square holes with side lengths ranging from 30 to $100 \mu \mathrm{m}$ were fabricated into the PR to expose the surface of the $\mathrm{Au}$ electrode by photolithography. Next, a self-assembled monolayer (SAM) of the photoswitchable molecules was deposited on the exposed Au surfaces. The molecule used in this study was the diarylethene species the chemical structure of which is shown in Figure 1(c). It has been chosen out of the large class of diarylethene molecules because furane-based diarylethene single-molecule junctions are known to have two different electrical conductance states, which are identified as the open state (low electrical conductance) or the closed (high electrical conductance) state. ${ }^{[34]}$ However, they have never been tested in monolayer-based molecular devices before. Furane-based diarylethenes are possible to be converted from the open to the closed state by exposing the molecule to UV light (at wavelengths from $300-350 \mathrm{~nm}$ ) or from the closed to the open state with visible light (at wavelengths from 570-630 nm). ${ }^{[34,37,38]}$ Furthmore, phenylene ethynylene-based molecules, featuring rather long conjugated arms bridging between the switching core and the anchoring groups, have been found to have 


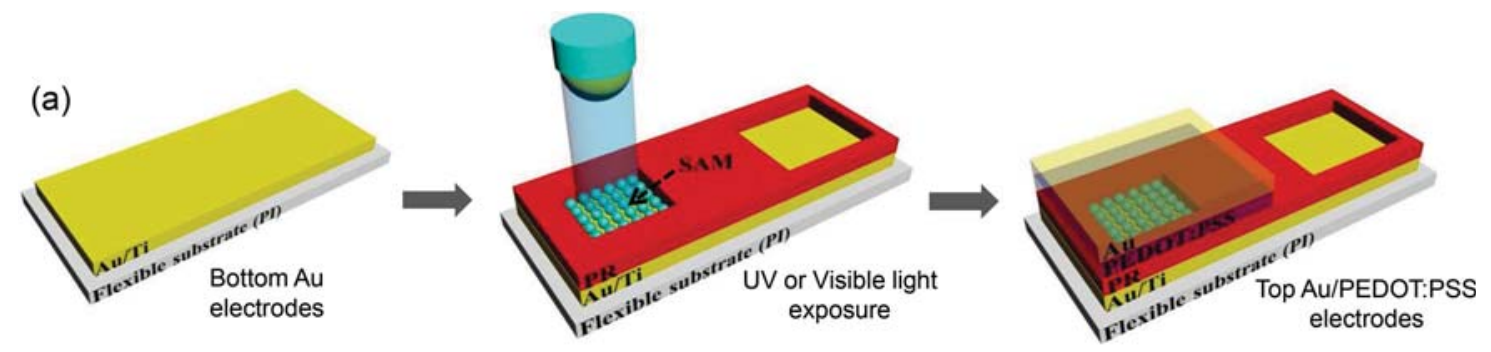

(b)

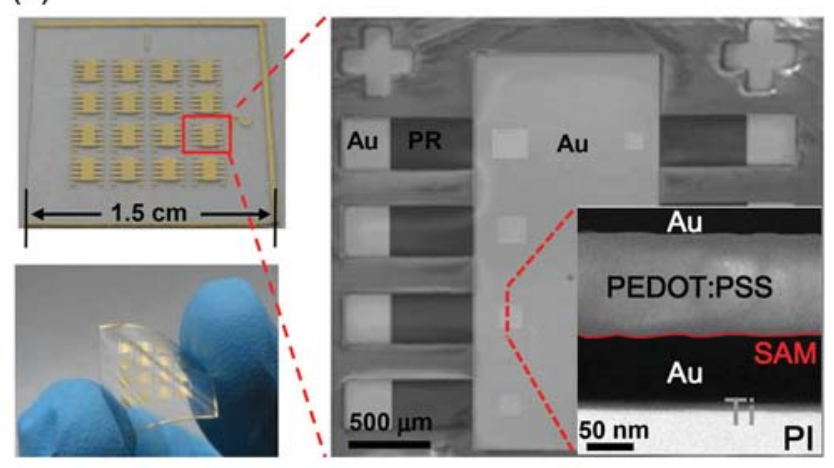

(c)

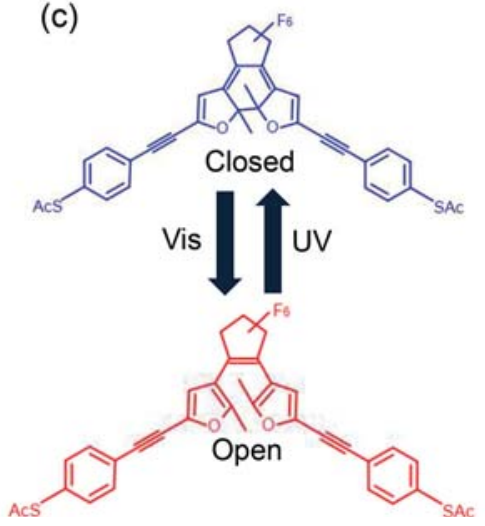

Figure 1. (a) Schematic illustration of the fabrication processes for the diarylethene molecular devices on a flexible substrate (PI substrate). (b) Optical, SEM, and cross-sectional TEM images of the fabricated diarylethene molecular devices (the molecular junctions are squares with holes possessing a side length ranging from 30 to $100 \mu \mathrm{m}$ ). (c) The chemical structure of the diarylethene molecule, which can be converted from an open (low conductance) state to a closed (high conductance) state by exposure to UV and visible light, respectively.

high quantum yields for both switching directions when investigated in solution. ${ }^{[39]}$ For the SAM deposition process, we prepared a diluted solution of the molecules $(\sim 3 \mathrm{mM})$ in ethanol and added a small amount of ammonium hydroxide $\left(\mathrm{NH}_{4} \mathrm{OH}\right)$ to the molecular solution to de-protect the acetyl $\left(\mathrm{COCH}_{3}\right.$, denoted as Ac) group from the thiol end-group for enabling electrical contract to the electrodes. ${ }^{[40-42]}$ Then, we immersed the samples in this molecular solution for 1-2 days in an $\mathrm{N}_{2^{-}}$ filled glove box. After finishing the SAM deposition, the samples were exposed to UV handed lamp capable of generating $312 \mathrm{~nm}$ UV light or visible-light lamp (fluorescent lamp) in a dark room for 1 hour to induce the closed or open states of the molecules, respectively. To fabricate the molecular devices, we used a conducting polymer, PEDOT:PSS ((poly-(3,4-ethylenedioxythiophene) stabilized with poly-(4-styrenesulfonic acid)) in a process that was first introduced by Akkerman et al.[15,16] The device yield for a simple metal/molecule/metal junction, without PEDOT:PSS was found to be as low as $\sim 1 \% .{ }^{[13,14]}$ The PEDOT:PSS layer serves as an interlayer electrode between the molecular layer and the metal and minimizes the electrical shorting problem. ${ }^{[15,16,18]}$ In this study, we observed device yields of $\sim 50 \%$ from the molecular devices fabricated with a PEDOT:PSS layer (100 nm thick). Then, we deposited the $\mathrm{Au}$ top electrode (20 $\mathrm{nm}$ thick) on top of the PEDOT:PSS layer. Figure 1(b) shows a series of optical, scanning electron microscopy (SEM), and cross-sectional transmission electron microscope (TEM) images of the fabricated molecular devices on the flexible substrates. More detailed device fabrication procedures are explained in the Experimental Section and in the Supporting Information (see Figure S1).
Figure 2(a) shows the current density-voltage $(J-V)$ data from the diarylethene molecular devices in the open and closed states under a flat substrate condition (defined as having a bending radius $=\infty$ ). We analyzed at least 20 devices in each open and closed state. The error bars in this plot are the standard deviation. We determined that the current observed in the closed state was higher than that from the open state by an order of magnitude, as has been found earlier for similar devices from thiophene-based diarylethenes on $\mathrm{Si}$ based rigid substrates. ${ }^{[6]}$ The high current density $\left(\sim 10^{6} \mathrm{~A} / \mathrm{m}^{2}\right.$ at $0.8 \mathrm{~V}$ ) observed in our devices reflects the SAM formation with our novel high-yield, flexible-substrate process. The electrical conductance discrepancy has been understood to arise from the change in the $\pi$-conjugation along the molecules when switching the ring between close and open states (see Figure $1(\mathrm{c})) \cdot{ }^{[6,34,37,38,43]}$ When the open state of diarylethene is exposed to UV light, the open state closes the central ring leading to a completely $\pi$-conjugated current path along the molecule. In contrast, when the closed state of diarylethene is exposed to visible light, the closed state breaks the current path into two rather decoupled systems. The $\pi$-system of closed state of diarylethene leads to a higher conductance than the broken $\pi$-system of open state of diarylethene. In addition, the metal-molecule contact (Au-thiol) of the closed state of diarylethene was found to give rise to a stronger broadening of the current-carrying molecular orbital than that of the open state, which also signals the stronger conjugation in the closed state. ${ }^{[34]}$

Additionally, we investigated the retention properties, i.e., the stability of the electrical characteristics of the diarylethene 
(a)

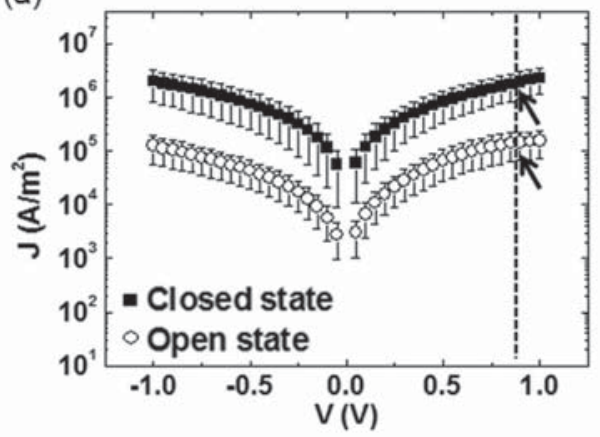

(c)

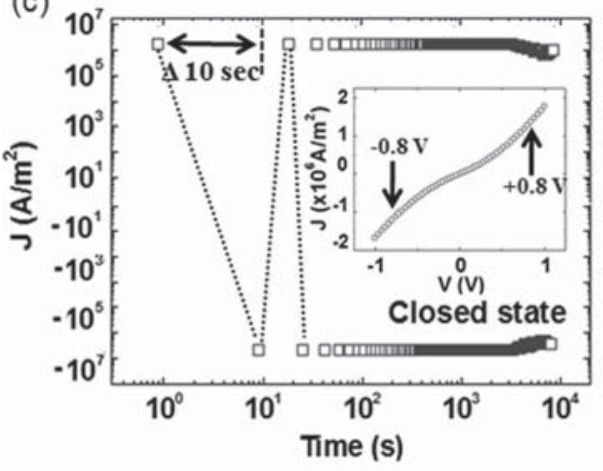

(b)

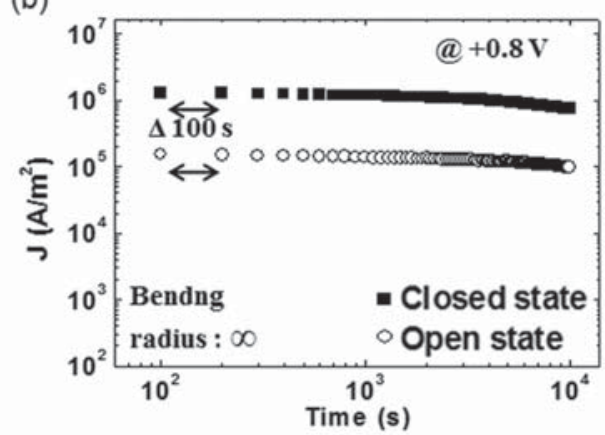

(d)

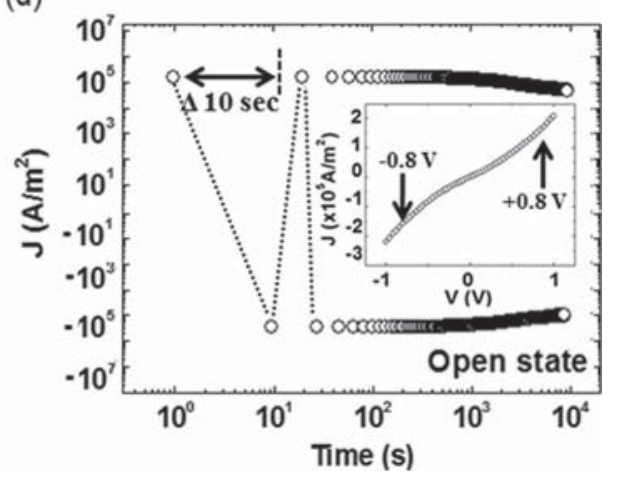

Figure 2. (a) J-V curves for the closed and open state diarylethene molecular devices on a flexible substrate under flat conditions. Colored arrows indicate the current density measured at $0.8 \mathrm{~V}$ (shown by a dashed line). (b) Retention characteristics for the closed and open states showing I values measured at $0.8 \mathrm{~V}$ for up to $10^{4}$ seconds at an increment of every 100 seconds. (c, d) Endurance characteristics of (c) the closed and (d) open states observed under two step voltage conditions. The current density values of the closed and open states were measured at $\pm 0.8 \mathrm{~V}$ for up to $10^{4}$ seconds at an increment of 10 seconds. The inset plots in $(c, d)$ show the $J-V$ curves prior to this measurement for the closed and open states.

molecular devices under a flat substrate condition by measuring the current density at $0.8 \mathrm{~V}$ over a long period, as shown in Figure 2(b). Specifically, the current density was measured in the open and closed states for up to $10^{4}$ seconds at an increment of 100 seconds. The current density of each state and the discrepancy between the two states were well maintained. This result indicates that both of the molecular device states (open and closed states) did not degrade when subjected to repeated voltage stresses.

We also investigated the endurance stability of the open and closed states by measuring the current density at alternating voltages of $\pm 0.8 \mathrm{~V}$ for up to $10^{4}$ seconds at an increment of 10 seconds. Figures 2(c) and 2(d) show the current density results on a logarithmic scale for the closed and open states, respectively. The insets of Figures 2(c) and 2(d) show the $J-V$ curves before the endurance test for the closed and open states, respectively. The current density was observed to be almost constant without any significant degradation. These results demonstrate that the diarylethene molecular device has excellent operational stability. We also measured the $J-V$ curves of the open and closed states after the devices were stored for 30 days under ambient conditions. In these measurements, we did not observe any noticeable degradation (see Figure S2 in the Supporting Information). All these results (Figure 2 and Figure S2) indicate that the devices retain their electrical properties well, demonstrating the reliability of diarylethene molecular devices in both photoswitching states.
To further test the robustness of these devices in view of applications making use of the flexibility of the device, we examined the electrical characteristics of the open and closed states when the device is subjected to mechanical bending. Figure 3(a) displays the current densities for the two states measured at $0.8 \mathrm{~V}$ under various bending configurations (bending radii equal to $\infty$ (flat), $10 \mathrm{~mm}, 5 \mathrm{~mm}$, and $1 \mathrm{~mm}$ ). The complete $J-V$ data sets acquired from these bending tests are provided in the Supporting Information (Figure S3). The results show that the electrical characteristics of both the open and closed states do not suffer any significant deterioration during mechanical stressing, and the current density was similar to that measured for the flat substrate conditions (Figure 2) with an order of magnitude difference between the two states regardless of the bending radius configuration.

The currents from the two states were also measured while being subjected to up to $10^{3}$ bending cycles, as shown in Figure $3(\mathrm{~b})$. The complete $J-V$ data sets and selected photos of our automatic cyclical bending test machine are provided in the Supporting Information (Figures S3 and S4). From these results, we also confirmed that the electrical characteristics of the molecular devices are consistent when subjected to repeated mechanical stresses. Figure 3(c) shows a device wound around a cylindrical bar with bending radius of $1 \mathrm{~mm}$. When the retention properties of the device in this bending condition were investigated, it was found that the currents for the open and closed states were consistent for up to $10^{4}$ seconds with an 

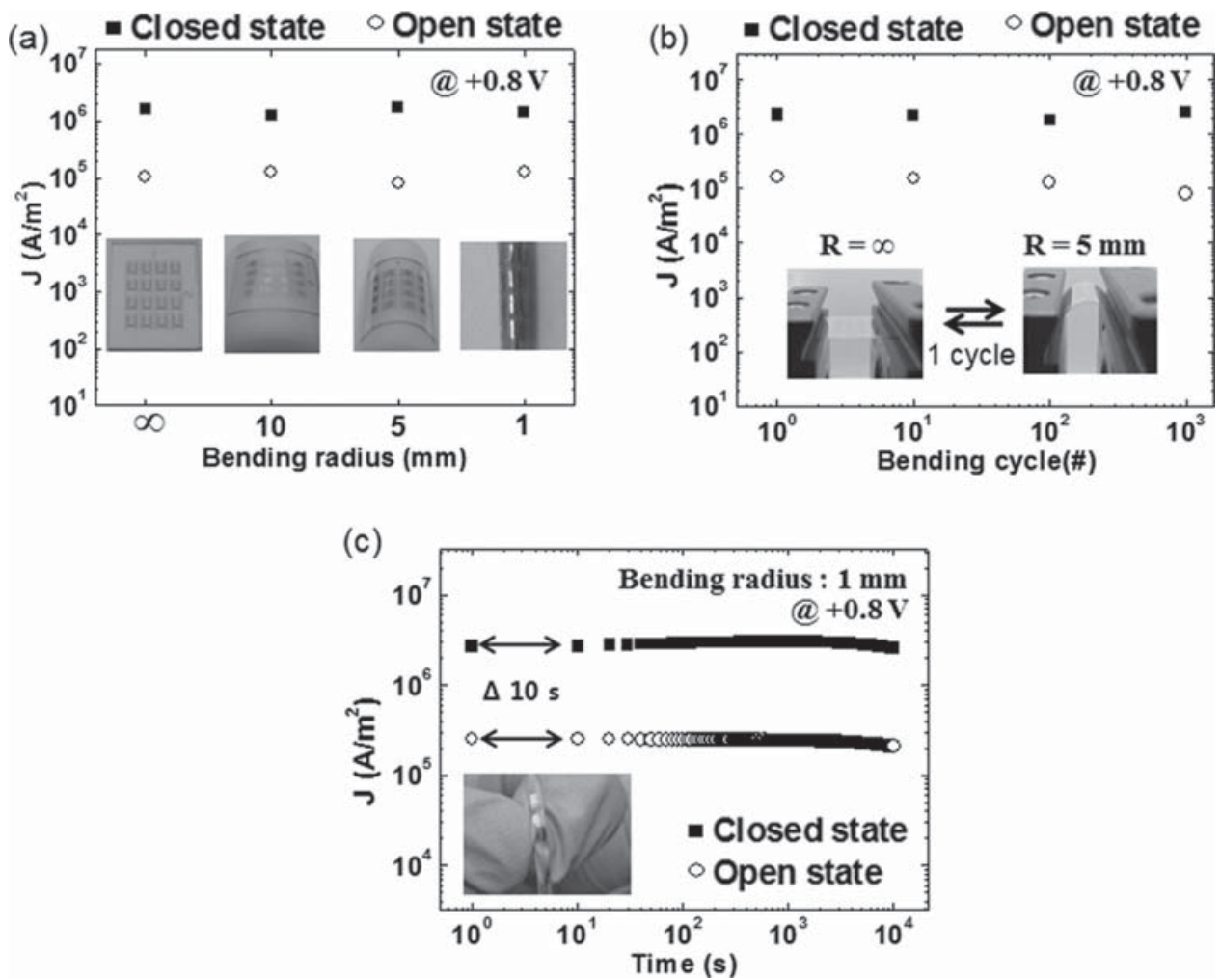

Figure 3. (a) J values measured at $0.8 \mathrm{~V}$ for closed and open states under flat substrate conditions (bending radius $=\infty$ ) and different bending conditions (bending radii $=10,5$, and $1 \mathrm{~mm}$ ). Inset photos show the molecular devices under different bending radius configurations. (b) I values measured at $0.8 \mathrm{~V}$ for the closed and open states after being subjected to repeated bending cycles $(0,10,100$, and 1000 times). Insets show one bending cycle at a bending radius $=5 \mathrm{~mm}$. (c) Retention characteristics for the closed and open states under severe bending conditions (bending radius $=1 \mathrm{~mm}$ ). The inset shows a molecular device that was wound around a cylindrical rod with the bending radius of $1 \mathrm{~mm}$.

order of magnitude difference between the two states. The consistency of the properties for the device, when subjected to the other bending conditions (such as a bending radius of $5 \mathrm{~mm}$ ), is provided in the Supporting Information (Figure S5). All these results (Figure 3 and Figures S3 and S5) demonstrated that the flexible molecular-scale devices containing photoswitching diarylethene molecules were reliably fabricated and operated with excellent stability when subjected to various mechanical stresses and high temperature.

Lastly, we investigated the charge conduction mechanisms of the open and closed states by performing temperature-variable current density-voltage $(J-V-T)$ measurements under flat and bent substrate conditions. Figure 4(a) shows the $J$ - $V$ - $T$ results for the two states for a mechanical deformation bending radius of $5 \mathrm{~mm}$ at variable temperatures from 80 to $300 \mathrm{~K}$. Figure $4(\mathrm{~b})$ shows the Arrhenius plot (i.e., the current density versus the inverse of the temperature) created from the data shown in Figure 4(a). We observed that the current density for both states did not change noticeably when the temperatures varied under both the flat (detailed data for the flat substrate condition are shown in Figure S6 in the Supporting Information) and bent substrate conditions (Figure 4). These results suggest that the main electrical conduction mechanism for the diarylethene molecules is tunneling for both the open and closed states, regardless of any mechanical deformation. ${ }^{[43]}$

We also did the $J-V-T$ measurements under high temperature range. The $J-V$ data of the closed and open states of our flexible diarylethene molecular devices did not change noticeably even when the temperarute was elevated up to $400 \mathrm{~K}$ (see Figure S7 in the Supporting Information). However, the devices thermally degraded when we heated the devices at $190{ }^{\circ} \mathrm{C}$ for $10 \mathrm{~min}$ on a hot plate.

Note that our flexible diarylethene molecular devices were not optically switchable (i.e., they were not turned on and off with UV or visible light after devices were fabricated). Instead our devices exhibited and maintained the high or low electrical conductance states when they were properly treated with UV or visible light during the fabrication, respectively. This lack of optical photoswitchability may be related to the device structure, in particular, $\mathrm{Au}$ top electrode (20 nm thick) and PEDOT:PSS layer (100 nm thick) on top of diarylethene molecular layer. To make optically photoswitching molecular devices, a more optimized device structure and higher intensity light would be desired.

In summary, we have fabricated molecular-scale electronic devices with photoswitching diarylethene molecules on flexible substrates. The diarylethene molecular devices exhibited either high or low electrical conductance states when exposed to UV or visible light during fabrication, respectively. The conductance in both states are sufficiently high to be measured and differ by an order of magnitude making them easy to discriminate with standard electronics. The electrical properties of the diarylethene molecular devices were found to be constant under variable temperature, repeated voltage cycling, various 
(a)

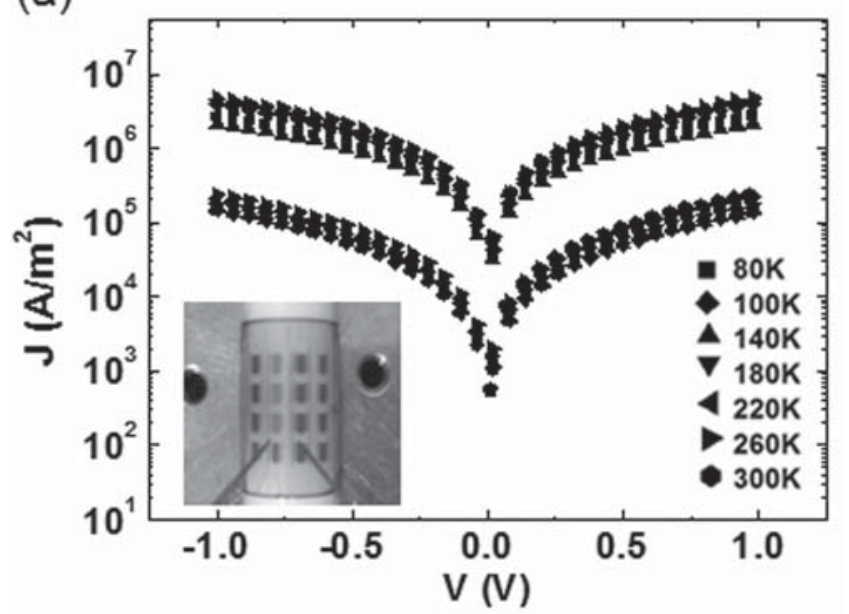

(b)

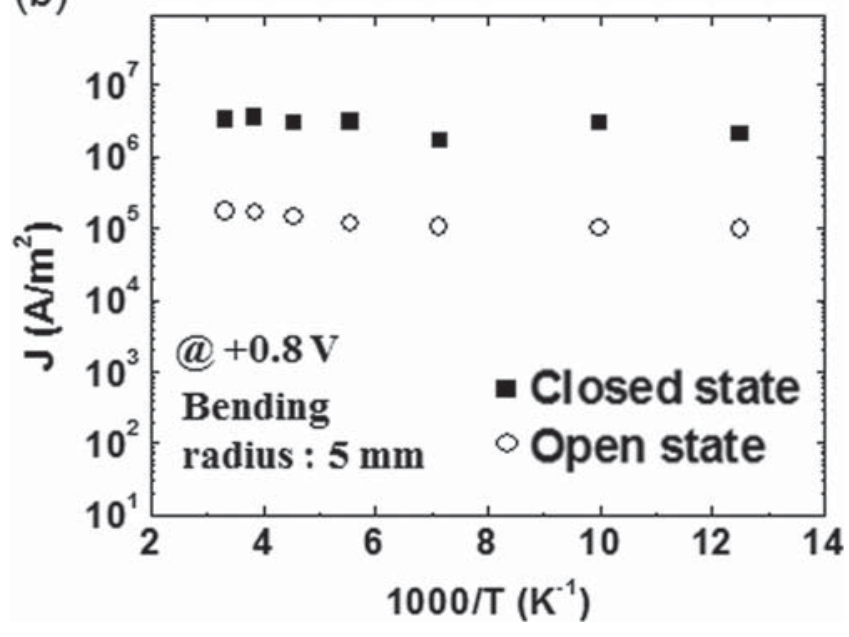

Figure 4. (a) Temperature-variable current density-voltage results for the closed and open states when subjected to bending (bending radius = $5 \mathrm{~mm}$ ). The inset shows a real device being measured. (b) Arrhenius plot of ) versus the inverse of the temperature (T) from the results shown in (a).

mechanical deformation configurations, including varying the bending radius (down to $1 \mathrm{~mm}$ ) and subjecting the substrates to repeated bending cycles, demonstrating that the molecular state as well as the contact geometry are robust. The current flowing in each state is dominated by a tunneling conduction mechanism regardless of any mechanical deformation. Our study provides a route way towards the fabrication and operation of optically or electrically functional molecules as flexible devices.

\section{Experimental Section}

Fabrication of Flexible Molecular Devices: The flexible substrates used in this study were first prepared by general cleaning process with isopropyl alcohol and deionized water. The patterned bottom Au $(60 \mathrm{~nm}) / \mathrm{Ti}(5$ $\mathrm{nm}$ ) electrodes were made on polyimide flexible substrates by using a shadow mask and an electron-beam evaporator. To create isolation wall, photoresist (Az5214E from Az Electronic Materials) was spincoated (4000 rpm for $1 \mathrm{~min}$ ) on the substrates, and then via-holes for diarylethene self-assembled monolayers (SAMs) were made. After that, in order to prevent the isolation wall from being soluble in SAM solution, the substrates were hard-baked at $190^{\circ} \mathrm{C}$ for $3 \mathrm{~h}$. The SAM deposition of diarylethene molecules was made by dipping the flexible substrates into the SAM solutions ( $\sim 3 \mathrm{mM}$ in anhydrous ethanol) in $\mathrm{N}_{2}$-filled glove box for 1-2 day. After deposition of SAMs, the substrates were rinsed with anhydrous ethanol and then dried by $\mathrm{N}_{2}$ stream in $\mathrm{N}_{2}$ filled glove box for $\sim 2 \mathrm{~h}$. Next, the flexible substrates were exposed to UV handed lamp or visible light lamp in a dark room for $\sim 1 \mathrm{~h}$ to determine the closed or open state of diarylethene molecules, respectively. Then, conducting polymer PEDOT:PSS (PH1000, purchased from Clevios ${ }^{\mathrm{TM}}$ ) layer was spin-coated (3500 rpm for 30 seconds) over the SAMs on substrates. After the PEDOT:PSS coating, Au $(20 \mathrm{~nm})$ top electrodes were deposited. Finally, with reactive ion etching (RIE) process (using $\mathrm{O}_{2}$ gas, $11 \mathrm{sccm}, 50 \mathrm{~W}$ ), residual PEDOT:PSS was removed.

Device Characterization: The electrical characteristics of the devices were measured with a semiconductor parameter analyzer (Keithley 4200 SCS) and a probe station system (JANIS Model ST-500).

\section{Supporting Information}

Supporting Information is available from the Wiley Online Library or from the author.

\section{Acknowledgements}

The authors appreciate the financial support of the National Creative Research Laboratory program (Grant No. 2012026372) and the National Core Research Center program (Grant No. R15-2008-006-03002-0) through the National Research Foundation of Korea (NRF) funded by the Korean Ministry of Science, ICT \& Future Planning. J.W., E.S., and T.H. thank the Deutsche Forschungsgemeinschaft for funding through SFB767.

[1] A. Aviram, M. A. Ratner, Chem. Phys. Lett. 1974, 29, 277.

[2] J. Chen, M. A. Reed, A. M. Rawlett, J. M. Tour, Science 1999, 286, 1550.

[3] W. J. Liang, M. P. Shores, M. Bockrath, J. R. Long, H. Park, Nature 2002, 417, 725.

[4] J. Park, A. N. Pasupathy, J. I. Goldsmith, C. Chang, Y. Yaish, J. R. Petta, M. Rinkoski, J. P. Sethna, H. D. Abruna, P. L. McEuen, D. C. Ralph, Nature 2002, 417, 722.

[5] J. E. Green, J. W. Choi, A. Boukai, Y. Bunimovich, E. Johnston-Halperin, E. Delonno, Y. Luo, B. A. Sheriff, K. Xu, Y. S. Shin, H. R. Tseng, J. F. Stoddart, J. R. Heath, Nature 2007, 445, 414.

[6] A. J. Kronemeijer, H. B. Akkerman, T. Kudernac, B. J. van Wees, B. L. Feringa, P. W. M. Blom, B. de Boer, Adv. Mater. 2008, 20, 1467.

[7] H. Song, Y. Kim, Y. H. Jang, H. Jeong, M. A. Reed, T. Lee, Nature 2009, 462, 1039.

[8] C. A. Nijhuis, W. F. Reus, A. C. Siegel, G. M. Whitesides, J. Am. Chem. Soc. 2011, 133, 15397.

[9] M. Del Valle, R. Gutierrez, C. Tejedor, G. Cuniberti, Nat. Nanotechnol. 2007, 2, 176.

[10] K. Matsuda, M. Irie, J. Am. Chem. Soc. 2001, 123, 9896. 
[11] S. J. van der Molen, J. Liao, T. Kudernac, J. S. Agustsson, L. Bernard M. Calame, B. J. van wees, B. L. Feringa, C. Schönenberger, Nano Lett. 2009, 9, 76.

[12] T. W. Kim, G. N. Wang, H. Lee, T. Lee, Nanotechnology 2007, 18, 315204.

[13] G. Wang, T. W. Kim, H. Lee, T. Lee, Phys. Rev. B 2007, 76, 205320

[14] G. Wang, H. Yoo, S. I. Na, B. Cho, D. Y. Kim, T. Lee, Thin Solid Films 2009, 518, 824.

[15] H. B. Akkerman, P. W. M. Blom, B. de Boer, Nature 2006, 441, 69

[16] P. A. Van Hal, E. C. P. Smits, T. C. T. Geuns, H. B. Akkerman, B. C. De Brito, S. Perissinotto, G. Lanzani, A. J. Kronemeijer, V. Geskinm, J. Cornil, P. W. Blom, B. de Boer, D. M. de Leeuw, Nat. Nanotechnol. 2008, 3, 7849.

[17] A. B. Neuhausen, A. Hosseini, J. A. Sulpizio, C. E. D. Chidsey, D. Goldhaber-Gordon, Acs Nano 2012, 6, 9920

[18] S. Park, G. Wang, B. Cho, Y. Kim, S. Song, Y. Ji, M. H. Yoon, T. Lee, Nat. Nanotechnol. 2012, 7, 438.

[19] G. Wang, Y Kim, M. Choe, T. W. Kim, T. Lee, Adv. Mater. 2011, 23 755.

[20] S. Seo, M. Min, J. Lee, T. Lee, S. Y. Choi, H. Lee, Angew. Chem. Int. Edit. 2012, 51, 108.

[21] S. Seo, M. Min, S. M. Lee, H. Lee, Nat. Commun. 2013, 4, 1920.

[22] X. D. Cui, X. Zarate, J. Tomfohr, O. F. Sankey, A. Primak, A. L. Moore, T. A. Moore, D. Gust, G. Harris, S. M. Lindsay, Nanotechnology 2002, 13, 5 .

[23] M. A. Rampi, G. M. Whitesides, Chem. Phys. 2002, 281, 373.

[24] V. B. Engelkes, J. M. Beebe, C. D. Frisbie, J. Am. Chem. Soc. 2004 $126,14287$.

[25] W. Wang, T. Lee, M. A. Reed, Phys. Rev. B 2003, 68, 035416.

[26] Y. Kim, H. Song, F. Strigl, H. F. Pernau, T. Lee, Phys. Rev. Lett 2011 106, 196804.

[27] J. M. Beebe, B. Kim, C. D. Frisbie, J. G. Kushmerick, Acs Nano. 2008, $2,827$.

[28] C. A. Nijhuis, W. F. Reus, G. M. Whitesides, J. Am. Chem. Soc 2009, 131, 17814.
[29] C. A. Nijhuis, W. F. Reus, J. R. Barber, M. D. Dickey, G. M. Whiteside, Nano Lett. 2010, 10,3611.

[30] C. A. Nijhuis, W. F. Reus, G. M. Whiteside, J. Am Chem. Soc. 2010, 132, 18386.

[31] N. Nerngchamnong, L. Yuan, D. C. Qi, D. Thompson, C. A. Nijhuis, Nat. Nanotechnol. 2013, 8, 113

[32] Y. Q. Liu, A. Offenhäusser, D. Mayer, Phys. Status Solidi A. 2010, 207, 891.

[33] H. Jeong, D. Kim, G. Wang, S. Park, H. Lee, K. Cho, W. T. Hwang, M. H. Yoon, Y. H. Jang, H. Song, D. Xiang, T. Lee, Adv. Funct. Mater 2013, in press; DOI: 10.1002/adfm.201303591

[34] Y. Kim, T. J. Hellmuth, D. Sysoiev, F. Pauly, T. Pietsch, J. Wolf, A. Erbe, T. Huhn, U. Groth, U. E. Steiner, E. Scheer, Nano Lett. 2012, 12, 3736.

[35] N. Katsonis, T. Kudernac, M. Walk, S. J. van der Molen, B. J. van Wees, B. L. Feringa, Adv. Mater. 2006, 18, 1397.

[36] D. Dulic, S. J. van der Molen, T. Kudernac, H. T. Jonkman, J. J. D. de Jong, T. N. Bowden, J. van Esch, B. L. Feringa, B. J. van Wees, Phys. Rev. Lett. 2003, 91, 207402.

[37] D. Sysoiev, A. Fedoseev, Y. Kim, T. E. Exner, J. Boneberg, T. Huhn, P. Leiderer, E. Scheer, U. Groth, U. E. Steiner, Chem Eur. J. 2011, 17, 6663.

[38] D. Sysoiev, T. Yushchenko, E. Scheer, U. Groth, U. E. Steiner, T. E. Exner, T. Huhn, Chem. Commun. 2012, 48, 11355.

[39] J. Wolf, I. Eberspächer, U. Groth, T. Huhn, J. Org. Chem. 2013, 78, 8366.

[40] J. M. Tour, L. JonesII, D. L. Pearson, J. J. S. Lamba, T. P. Burgin, G. M. Whitesides, D. L. Allara, A. N. Parkih, S. V. Atre, J. Am. Chem. Soc. 1995, 117, 9529.

[41] J. G. Kushmerick, J. Lazorcik, C. H. Patterson, R. Shashidhar, D. S. Seferos, G. C. Bazan, Nano Lett. 2004, 4, 639.

[42] H. Valkenier, E. H. Huisman, P. A. van Hal, D. M. de Leeuw, R. C. Chiechi, J. C. Hummelen, J. Am. Chem. Soc. 2011, 133, 4930.

[43] D. Dulic, T. Kudernac, A. Puzys, B. L. Feringa, B. J. van Wees, Adv. Mater. 2007, 19, 2898 